\title{
On beta-function of tube of light cone
}

\author{
YuRI A. NERETIN 1
}

We construct $B$-function of the Hermitian symmetric space $\mathrm{O}(n, 2) / \mathrm{O}(n) \times \mathrm{O}(2)$ or equivalently of the tube $\left(\operatorname{Re} z_{0}\right)^{2}>\left(\operatorname{Re} z_{1}\right)^{2}+\cdots+\left(\operatorname{Re} z_{n}\right)^{2}$ in $\mathbb{C}^{n+1}$.

\section{Formulation of the result}

1.1. Preliminary references. The beta-function of symmetric cones

$$
\operatorname{GL}(n, \mathbb{R}) / \mathrm{O}(n), \operatorname{GL}(n, \mathbb{C}) / \mathrm{U}(n), \operatorname{GL}(n, \mathbb{H}) / \mathrm{Sp}(n)
$$

was constructed by Gindikin in [4, see also 6]. For the remaining series of classical symmetric spaces the beta-function was obtained in [7]. The subseries $\mathrm{O}(n, 2) / \mathrm{O}(n) \times \mathrm{O}(2)$ has two beta-functions, the first one is a special case of the beta-function of $\mathrm{O}(p, q) / \mathrm{O}(p) \times \mathrm{O}(q)$. The second beta-function is discussed here, it is related to the Hermitian structure of these spaces 2

1.2. The tube of light cone. Consider the space $\mathbb{C}^{n+1}$ with coordinates $z_{0}, z_{1}, \ldots, z_{n}$. By $\mathfrak{T}_{n}$ we denote the tube

$$
\left(\operatorname{Re} z_{0}\right)^{2}>\left(\operatorname{Re} z_{1}\right)^{2}+\left(\operatorname{Re} z_{2}\right)^{2}+\cdots+\left(\operatorname{Re} z_{n}\right)^{2}, \quad \operatorname{Re} z_{0}>0
$$

The space $\mathfrak{T}_{n}$ is homogeneous with respect to the pseudo-orthogonal group $\mathrm{O}(n+1,2)$ (apparently, this was discovered by E.Cartan, 2], for a discussion, see, for instance, [5, [10, 6]), namely

$$
\mathfrak{T}_{n} \simeq \mathrm{O}(n+1,2) / \mathrm{O}(n+1) \times \mathrm{O}(2)
$$

This group acts on $\mathfrak{T}$ by quadratic-fractional transformations and is generated by the maps of the following 3 types

a) Shifts $z \mapsto z+i a$, where $a \in \mathbb{R}^{n+1}$.

b) Linear transformations $z \mapsto z g$, where $g \in \mathrm{SO}_{0}(n, 1)$, i.e., $g$ is a real matrix preserving the quadratic form $x_{0}^{2}-x_{1}^{2}-\cdots-x_{n}^{2}$.

c) Quasiinversion $z \mapsto z /(z, z)$, where $(z, z):=z_{0}^{2}-z_{1}^{2}-\cdots-z_{n}^{2}$.

\footnotetext{
${ }^{1}$ Supported by the grant FWF, project P19064, Russian Federal Agency for Nuclear Energy, grant NWO.047.017.015, and grant JSPS-RFBR-07.01.91209

${ }^{2}$ This note is some kind of an addendum to my paper [7] on matrix beta-functions; originally it was an omitted section of the last work. Since the Plancherel formula for Berezin representations (which was my actual purpose) for $\mathrm{O}(n, 2) / \mathrm{O}(n) \times \mathrm{O}(2)$ was known due Berezin himself [1] (proof was published by Unterberger and Upmeier [1]) I omitted 6-parameter integral (1.1). However, after a discussion with prof. G.Roos I understood that this integral can be interesting by itself since this series of symmetric spaces is familiar to various non-representation-theoretic people.
} 
This space has numerous names, in particular, Cartan domain of 4-th type, future tube, Lie spheres, Lie ball 3 .

We prefer another realization of the same space. Namely, consider $\mathbb{C}^{n+1}$ with coordinates $u_{1}, u_{2}, z_{1}, \ldots, z_{n-1}$,

$$
\begin{array}{r}
u_{1}=v_{1}+i w_{1} ; \\
u_{2}=v_{2}+i w_{2} ; \\
z_{j}:=x_{j}+y_{j}
\end{array}
$$

We define our tube $\mathfrak{T}_{n}$ by the inequalities

$$
v_{1} v_{2}-\sum x_{j}^{2}>0, \quad v_{1}>0
$$

1.3. Formulation of the result. For a nonzero complex $a$ and complex $\lambda, \mu$ denote

$$
a^{\{\lambda \mid \mu\}}:=a^{\lambda} \bar{a}^{\mu}
$$

We also denote

$d u_{1}:=d v_{1} d w_{1}, \quad d z_{j}:=d x_{j} d y_{j}, \quad d u:=d u_{1} d u_{2}, \quad d z:=d x_{1} d y_{1} \ldots d x_{n-1} d y_{n-1}$

In particular $d u d z$ denotes an integration with respect to the Lebesgue measure on $\mathfrak{T}_{n}$.

Our purpose is the following formula

$$
\begin{array}{r}
\int_{\mathfrak{T}_{n}} \frac{v_{1}^{\lambda_{1}-\lambda_{2}}\left(v_{1} v_{2}-\sum x_{j}^{2}\right)^{\lambda_{2}-n-1} d u d z}{\left(1+u_{1}\right)^{\left\{\sigma_{1}-\sigma_{2} \mid \tau_{1}-\tau_{2}\right\}}\left(\left(1+u_{1}\right)\left(1+u_{2}\right)-\sum z_{j}^{2}\right)^{\left\{\sigma_{2} \mid \tau_{2}\right\}}}= \\
=2^{1-\sigma_{1}-\tau_{1}+n} \pi \frac{\Gamma\left(\lambda_{1}-\frac{1}{2}(n+1)\right) \Gamma\left(\sigma_{1}+\tau_{1}-\lambda_{1}-\frac{1}{2}(n-1)\right)}{\Gamma\left(\sigma_{1}-\frac{1}{2}(n-1)\right) \Gamma\left(\tau_{1}-\frac{1}{2}(n-1)\right)} \times \\
\times 2^{2-\sigma_{2}-\tau_{2}+n} \pi^{n} \frac{\Gamma\left(\lambda_{2}-n\right) \Gamma\left(\sigma_{2}+\tau_{2}-\lambda_{2}\right)}{\Gamma\left(\sigma_{2}\right) \Gamma\left(\tau_{2}\right)}
\end{array}
$$

where $\lambda_{1}, \lambda_{2}, \sigma_{1}, \sigma_{2}, \tau_{1}, \tau_{2} \in \mathbb{C}$.

REMARK. Let us explain the meaning of complex powers. The base numbers $v_{1}, v_{1} v_{2}-\sum x_{j}^{2}$ of the numerator are positive reals. Next, the point $u_{1}=1$, $u_{2}=1, z=0$ is contained in $\mathfrak{T}_{n}$. The denominator is well defined at this point.

\footnotetext{
${ }^{3}$ The last two terms arose as a result of non-correct successive translations German-ChinesEnglish-Russian-English.

Initially, a 'Lie sphere' was an oriented subsphere in $S^{n}=\mathbb{R}^{n} \cup \infty$ or a point. Lie spheres was the space of Lie spheres; It is a homogeneous space $\mathrm{O}(n+2) / \mathrm{O}(n+2) \times \mathrm{O}(2)$. Lie sphere geometry, see for instance [3, was a geometry of this space. Hua Loo Keng extended the term 'Lie spheres' to the dual symmetric space $\mathrm{O}(n+2,2) / \mathrm{O}(n+2) \times \mathrm{O}(2)$.

In Russian edition of Hua's book [5] the space $\mathrm{O}(n, 2) / \mathrm{O}(n) \times \mathrm{O}(2)$ became a Lie sphere ('sfera Li'; in fact there are no traces of the original meaning of 'Lie spheres' in Hua's book). However, the term 'sphere' for an open domain is too peculiar and our space turned into a 'Lie ball' in the English edition.
} 
Since the domain $\mathfrak{T}_{n}$ is simply connected, the corresponding branches of the power functions are well defined.

1.4. Meaning of factors. a) Functions $v_{1}^{\lambda_{1}-\lambda_{2}}\left(v_{1} v_{2}-\sum x_{j}^{2}\right)^{\lambda_{2}-n-1}$ are precisely the eigenfunctions of the parabolic subgroup in $\mathrm{O}(n+1,2)$. Also $\left(v_{1} v_{2}-\sum x_{j}^{2}\right)^{-n-1}$ is the density of the $\mathrm{O}(n+1,2)$-invariant measure on $\mathfrak{T}_{n}$.

b) $\left(\left(1+u_{1}\right)\left(1+u_{2}\right)-\sum z_{j}^{2}\right)$ is the standard term that is present in formulae for the Cauchy kernel, the Bargman kernel, see [5] and (more generally) for the Berezin kernels on $\mathfrak{T}_{n}$.

1.5. Comments. Special cases. a) If $\lambda_{1}=\lambda_{2}=0, \sigma_{1}=\tau_{1}=0, \sigma_{2}=\tau_{2}$, then we get one of Hua integrals, [5]

b) The Plancherel formula for Berezin kernels for the spaces $\mathrm{O}(n+1,2) / \mathrm{O}(n+$ 1) $\times \mathrm{O}(2)$ is reduced to our integral with $\sigma_{1}=\tau_{1}=0, \sigma_{2}=\tau_{2}$ (see [11]). Apparently, Berezin himself (he perished in an accident in 1980) derived this formula in some another way (however, his proof is unknown; see also [11).

c) For $n=3,4,6$ there are the following exceptional isomorphisms of homogeneous spaces

$$
\begin{aligned}
& \mathrm{O}(3,2) / \mathrm{O}(3) \times \mathrm{O}(2)=\mathrm{Sp}(6, \mathbb{R}) / \mathrm{U}(2) \\
& \mathrm{O}(4,2) / \mathrm{O}(4) \times \mathrm{O}(2)=\mathrm{U}(2,2) / \mathrm{U}(2) \times \mathrm{U}(2) \\
& \mathrm{O}(6,2) / \mathrm{O}(6) \times \mathrm{O}(2)=\mathrm{SO}^{*}(8) / \mathrm{U}(4)
\end{aligned}
$$

In these cases, our integrals coincide with matrix beta-integrals obtained in [7].

\section{Calculations}

2.1. Change of variables. Firstly, we transform our integral to

$$
\int_{v_{1}>0, w_{1} \in \mathbb{R}} \frac{v_{1}^{\lambda_{1}-n-1}}{\left(1+u_{1}\right)^{\left\{\sigma_{1} \mid \tau_{1}\right\}}} \cdot\left\{\int_{v_{2}-\frac{1}{v_{1}} \sum x_{j}^{2}>0} \frac{\left(v_{2}-\frac{1}{v_{1}} \sum x_{j}^{2}\right)^{\lambda_{2}-n-1} d u_{2} d z}{\left(1+u_{2}-\frac{1}{1+u_{1}} \sum z_{j}^{2}\right)^{\left\{\sigma_{2} \mid \tau_{2}\right\}}}\right\} d u_{1}
$$

Next, we change the variable $v_{2}$ by $r$,

$$
r:=v_{2}-\frac{1}{v_{1}} \sum x_{j}^{2}
$$

(the Jacobian of this substitution $=1$ ). The interior integral now is reduced to

$$
\int_{r>0, w_{1} \in \mathbb{R}, x \in \mathbb{R}^{n-1}, y \in \mathbb{R}^{n-1}} \frac{r^{\lambda_{2}-n-1} d r d w_{2} d x d y}{\left(1+r+i w_{2}+\frac{1}{v_{1}} \sum x_{j}^{2}-\frac{1}{1+u_{1}} \sum z_{j}^{2}\right)^{\left\{\sigma_{2} \mid \tau_{2}\right\}}}
$$

2.2. Next change of variables. Now we wish to decompose the expression

$$
H:=1+r+i w_{1}+\frac{1}{v_{1}} \sum x_{j}^{2}-\frac{1}{1+u_{1}} \sum z_{j}^{2}
$$


as a sum of imaginary and real parts. For this purpose, we write

$\operatorname{Re} \frac{1}{1+u_{1}} \sum z_{j}^{2}=\operatorname{Re} \frac{\sum\left(x_{j}^{2}-y_{j}^{2}+2 i x_{j} y_{j}\right)}{1+v_{1}+i w_{1}}=\sum_{j} \frac{\left(x_{j}^{2}-y_{j}^{2}\right)\left(1+v_{1}\right)+2 x_{j} y_{j} w_{1}}{\left(1+v_{1}\right)^{2}+w_{1}^{2}}$

Therefore,

$$
\operatorname{Re} H=1+r+\sum_{j}\left(\begin{array}{ll}
x_{j} & y_{j}
\end{array}\right) S\left(\begin{array}{l}
x_{j} \\
y_{j}
\end{array}\right)
$$

where

$$
S:=\left(\begin{array}{cc}
\frac{1}{v_{1}}-\frac{1+v_{1}}{\left(1+v_{1}\right)^{2}+w_{1}^{2}} & \frac{-w_{1}}{\left(1+v_{1}\right)^{2}+w_{1}^{2}} \\
\frac{-w_{1}}{\left(1+v_{1}\right)^{2}+w_{1}^{2}} & \frac{1+v_{1}}{\left(1+v_{1}\right)^{2}+w_{1}^{2}}
\end{array}\right)
$$

Note that,

$$
\operatorname{det} S=\frac{1}{v_{1}\left(\left(1+v_{1}\right)^{2}+w_{1}^{2}\right)}=v_{1}^{-1}\left(1+v_{1}+i w_{1}\right)^{-\{1 \mid 1\}}=v_{1}^{-1}\left(1+u_{1}\right)^{-\{1 \mid 1\}}
$$

Thus, det $S>0$, the diagonal elements of $S$ also are positive. Hence $S$ is positive definite; therefore $S^{1 / 2}$ is well defined.

Our next change of variables is

$$
\left(\begin{array}{ll}
x_{j} & y_{j}
\end{array}\right)=\left(\begin{array}{ll}
p_{j} & q_{j}
\end{array}\right) S^{-1 / 2}
$$

Its Jacobian is

$$
v_{1}^{(n-1) / 2}\left(1+u_{1}\right)^{(n-1) / 2 \cdot\{1 \mid 1\}}
$$

Finally, the 'interior integral' (2.1) comes to the form

$$
\begin{aligned}
& v_{1}^{(n-1) / 2}\left(1+u_{1}\right)^{(n-1) / 2 \cdot\{1 \mid 1\}} \times \\
\times & \int_{r>0, w_{2} \in \mathbb{R}, p \in \mathbb{R}^{n-1}, q \in \mathbb{R}^{n-1}} \frac{r^{\lambda_{2}-n-1} d r d w_{2} d p d q}{\left(1+r+\sum\left(p_{j}^{2}+q_{j}^{2}\right)+i w_{2}+i Q\left(p, q, v_{1}, w_{1}\right)\right)^{\left\{\sigma_{2} \mid \tau_{2}\right\}}}
\end{aligned}
$$

where $Q\left(p, q, v_{1}, w_{1}\right)$ is a real expression (its explicit form is unessential for us).

2.3. Separation of variables. Now we change the variable $w_{2}$ to

$$
h:=w_{2}+Q(\cdot)
$$

The Jacobian is 1 , and we reduce our initial integral to the product

$$
\begin{aligned}
I \cdot J:= & \int_{v_{1}>0, w_{1} \in \mathbb{R}} \frac{v_{1}^{\lambda_{1}-n / 2-3 / 2} d v_{1} d w_{1}}{\left(1+v_{1}+i w_{1}\right)^{\left\{\sigma_{1}-(n-1) / 2 \mid \tau_{1}-(n-1) / 2\right\}}} \times \\
& \times \int_{r>0, h \in \mathbb{R}, p \in \mathbb{R}^{n-1}, q \in \mathbb{R}^{n-1}} \frac{r^{\lambda_{2}-n-1} d r d h d p d q}{\left(1+r+\sum\left(p_{j}^{2}+q_{j}^{2}\right)+i h\right)^{\left\{\sigma_{2} \mid \tau_{2}\right\}}}
\end{aligned}
$$

where $I$ denotes the first integral factor, and $J$ the second one. 
2.4. An auxiliary integral. First, we derive the identity

$$
\int_{x>0, y \in \mathbb{R}} \frac{x^{\alpha-1} d x d y}{(1+x+i y)^{\{\beta \mid \gamma\}}}=2^{2-\beta-\gamma} \pi \frac{\Gamma(\alpha) \Gamma(\beta+\gamma-\alpha-1)}{\Gamma(\beta) \Gamma(\gamma)}
$$

We represent the left-hand side as

$$
\int_{0}^{\infty} d v \cdot v^{\alpha-1} \int_{-\infty}^{\infty} \frac{d w}{(1+v+i w)^{\{\beta \mid \gamma\}}}
$$

The interior integral is the Cauchy beta-integral (see [9] 2.2.6.31) and we get

$$
2 \pi \cdot 2^{1-\beta-\gamma} \frac{\Gamma(\beta+\gamma-1)}{\Gamma(\beta) \Gamma(\gamma)} \int_{0}^{\infty} \frac{v^{\alpha-1} d v}{(1+v)^{\beta+\gamma-1}}
$$

The last integral is a rephrasing of the definition of the beta-function (see [9] 2.2.4.29). We get

$$
2 \pi \cdot 2^{1-\beta-\gamma} \frac{\Gamma(\beta+\gamma-1)}{\Gamma(\beta) \Gamma(\gamma)} \cdot \frac{\Gamma(\alpha) \Gamma(\beta+\gamma-\alpha-1)}{\Gamma(\beta+\gamma-1)}
$$

\subsection{The first factor. By (2.3),}

$$
I:=2^{1-\sigma_{1}-\tau_{1}+n} \pi \frac{\Gamma\left(\lambda_{1}-\frac{1}{2}(n+1)\right) \Gamma\left(\sigma_{1}+\tau_{1}-\lambda_{1}-\frac{1}{2}(n-1)\right)}{\Gamma\left(\sigma_{1}-\frac{1}{2}(n-1)\right) \Gamma\left(\tau_{1}-\frac{1}{2}(n-1)\right)}
$$

2.6. The second factor. Now we evaluate the factor $J$ in (2.2). First, we pass to the spherical coordinates

$$
R^{2}=\sum\left(p_{j}^{2}+q_{j}^{2}\right)
$$

in $\mathbb{R}^{2 n-2}$. We get (see [9], 3.3.2.1)

$$
\frac{2 \pi^{n-1}}{\Gamma(n-1)} \int_{r>0, R>0, h \in \mathbb{R}} \frac{r^{\lambda_{2}-n-1} R^{2 n-3} d r d R d h}{\left(1+r+R^{2}+i h\right)^{\left\{\sigma_{2} \mid \tau_{2}\right\}}}
$$

Next, we substitute $\rho:=R^{2}$,

$$
J=\frac{\pi^{n-1}}{\Gamma(n-1)} \int_{r>0, R>0, h \in \mathbb{R}} \frac{r^{\lambda_{2}-n-1} \rho^{n-2} d r d \rho d h}{(1+r+\rho+i h)^{\left\{\sigma_{2} \mid \tau_{2}\right\}}}
$$

Next, we pass from the variables $(r, \rho)$ to

$$
(x, r):=(r+\rho, r)
$$

i.e.,

$$
J=\frac{\pi^{n-1}}{\Gamma(n-1)} \int_{x>0,0<r<x, h \in \mathbb{R}} \frac{r^{\lambda_{2}-n-1}(x-r)^{n-2} d r d x d h}{(1+x+i h)^{\left\{\sigma_{2} \mid \tau_{2}\right\}}}
$$


Now we integrate in $r$ using the standard definition of the beta-function,

$$
J=\frac{\pi^{n-1}}{\Gamma(n-1)} \mathrm{B}(\lambda-n, n-1) \int_{x>0, h \in \mathbb{R}} \frac{x^{\lambda_{2}-2} d x d h}{(1+x+i h)^{\left\{\sigma_{2} \mid \tau_{2}\right\}}}
$$

The last integral is of the form (2.3). Finally,

$$
J=2^{2-\sigma_{2}-\tau_{2}-n} \pi^{n} \frac{\Gamma\left(\lambda_{2}-n\right) \Gamma\left(\sigma_{2}+\tau_{2}-\lambda_{2}\right)}{\Gamma\left(\sigma_{2}\right) \Gamma\left(\tau_{2}\right)}
$$

\section{References}

[1] Berezin, F. A. The connection between covariant and contravariant symbols of operators on classical complex symmetric spaces. (Russian) Dokl. Akad. Nauk SSSR 241 (1978), no. 1, 15-17. English translation: Sov. Math. Dokl. 19 (1978), 786-789

[2] Cartan, E. Sur les domaines bornés homogenés de l'espace des $n$ variables complexes. (French) [J] Abhandl. Hamburg 11, 116-162. Published: 1935

[3] Cecil, Th. E.Lie sphere geometry. With applications to submanifolds. Springer-Verlag, New York, 1992.

[4] Gindikin, S. G. Analysis in homogeneous domains. (Russian) Uspehi Mat. Nauk 191964 no. 4 (118), 3-92.

[5] Hua, L. K. Harmonic analysis of functions of several complex variables in the classical domains. (Chinese), Science Press, Peking, 1958. Russian edition: Izdat. Inostr. Lit., Moscow 1959 (translated by M. A. Evgrafov; edited by M. I. Graev). English edition: American Mathematical Society, Providence, R.I. 1963 (translated by L.Ebner and A.Koranyi).

[6] Faraut, J.; Koranyi, A. Analysis on symmetric cones. Oxford: Clarendon Press. xii, 382 p. (1994)

[7] Neretin, Yu.A. Matrix analogues of the beta function and Plancherel's formula for Berezin kernel representations. Sb. Math. 191, No.5, 683-715 (2000); translation from Mat. Sb. 191, No.5, 67-100 (2000).

[8] Neretin, Yu. A. Plancherel formula for Berezin deformation of $L^{2}$ on Riemannian symmetric space. J. Funct. Anal. 189, No.2, 336-408 (2002).

[9] Prudnikov, A. P.; Brychkov, Yu. A.; Marichev, O. I. Integrals and series. Vol. 1. Elementary functions. Translated from the Russian. Gordon \& Breach Science Publishers, New York, 1986.

[10] Pjateckiı̌-Šapiro, I. I. Geometry of classical domains and theory of automorphic functions Gosudarstv. Izdat. Fiz.-Mat. Lit., Moscow 1961 
[11] Unterberger, A., Upmeier, H., The Berezin transform and invariant differential operators. Comm.Math.Phys.,164, 563-597(1994)

Math.Dept., University of Vienna,

Nordbergstrasse, 15, Vienna, Austria

$\&$

Institute for Theoretical and Experimental Physics,

Bolshaya Cheremushkinskaya, 25, Moscow 117259, Russia

e-mail: neretin(at) mccme.ru

URL : www . mat . univie.ac . at/ neretin 